\title{
On the Relationship between Fuzzy Subgroups and Indistinguishability Operators
}

\author{
D. Boixader \& J. Recasens \\ Secció Matemàtiques i Informàtica \\ ETS Arquitectura del Vallès \\ Universitat Politècnica de Catalunya \\ Pere Serra 1-15 \\ 08190 Sant Cugat del Vallès \\ Spain
}

\begin{abstract}
Fuzzy subgroups are revisited considering their close relationship with indistinguishability operators (fuzzy equivalences) invariant under translations.

Different ways to obtain new fuzzy subgroups from a given one are provided and different ways to characterize normal fuzzy subgroups are obtained.

The idea of double coset of two (crisp) subgroups allow us to relate them via their equivalence classes. This is generalized to the fuzzy framework.

The conditions in which a fuzzy relation $R$ on a group $G$ can be considered a fuzzy subgroup of $G \times G$ are obtained.

Keywords: fuzzy subgroup, normal fuzzy subgroup, $T$-indistinguishability operator, invariance under translations, double coset.
\end{abstract}

\section{Introduction}

Indistinguishability operators, also called fuzzy equivalence relations or fuzzy equalities, fuzzify the concepts of crisp equivalence relation and crisp equality. 
They were introduced by Zadeh in [15] and there is a big amount of literature dealing with them.

The natural generalization of crisp subgroup of a group $(G, \circ)$ to the fuzzy framework was given by Rosenfeld [13] using the minimum t-norm for modelling closeness and many works have been devoted to the topic. One of the most interesting properties of these fuzzy subgroups is that their alpha-cuts are crisp subgroups, a result intimately related to the fact that the alpha-cuts of a min-indistinguishability operator on a universe $X$ are partitions of $X$. Lately, fuzzy subgroups with respect to general t-norms have been considered and studied $[2,5,11]$.

To every fuzzy subgroup $\mu$ of a group $(G, \circ)$ two indistinguishability operators $E_{\mu}$ and ${ }_{\mu} E$ can be associated that are left and a right invariant under translations respectively. If the fuzzy subgroup $\mu$ is normal, then the left and right indistinguishability operators $E_{\mu}$ and ${ }_{\mu} E$ coincide and the operation of the group is compatible with them.

To mention only one field where invariance under translations is usually assumed and needed, we can consider Fuzzy Mathematical Morphology [3]. In the study of objects in the plane $\mathbb{R}^{2}$, isotropy is usually assumed and hence the used relations must be invariant under translations. For one of these relations $R$, two of the basic operators, dilation $D$ and erosion $E$, can be defined by $D(\mu)(\vec{x})=\sup _{\vec{y} \in \mathbb{R}^{2}} T(R(\vec{y}-\vec{x}), \mu(\vec{y}))$ and $E(\mu)(\vec{x})=\inf _{\vec{y} \in \mathbb{R}^{2}} \vec{T}(\mu(\vec{y}) \mid R(\vec{y}-\vec{x}))$. If $R$ is an indistinguishability operator, then the structural element is the fuzzy subgroup $R((0,0), \cdot)$ of $\mathbb{R}^{2}$.

The study of the closed relation between fuzzy subgroups and indistinguishability operators will be continued in this paper. After a Section 2 of basic or known results needed to make the paper as self contained as possible, Section 3 will focus on different ways to obtain new fuzzy subgroups from a given fuzzy subgroup $\mu$ and on the relation between the indistinguishability operators associated to these new fuzzy subgroups and the ones associated to $\mu$. Being normality of a fuzzy subgroup such important, several characterizations of this property are obtained.

A fuzzy subgroup $\mu$ of $G$, considered as a fuzzy subset of $G$, generates an indistinguishability operator $E^{\mu}[14,11]$. Section 4 is devoted to the relationship between $E^{\mu}$ and the right and left invariant indistinguishability operators associated to $\mu$.

Double cosets are a way to relate two subgroups. In Section 5 the concept of double coset is fuzzified and the indistinguishability operators having 
double cosets as columns are studied.

A fuzzy relation $R$ on a group $G$ is a fuzzy subset of $G \times G$ and therefore a candidate to be a fuzzy subgroup of $G \times G$. This will be studied in Section 6 where conditions on $R$ to be a fuzzy subgroup are given.

In the section of Concluding Remarks, some interesting examples of the real line and the plane will be analyzed.

\section{Preliminaries}

This section contains some definitions and properties related to fuzzy subgroups, indistinguishability operators and their connection that will be needed later on. All the results are known and the reader is referred to $[5,7,10,11]$ for comments and proofs.

Throughout the paper $T$ will denote a given t-norm.

Fuzzy subgroups were introduced by Rosenfeld [13] as a natural generalization of the concept of subgroup and have been widely studied [10].

Definition 2.1. Let $(G, \circ)$ be a group, e its identity element and $\mu$ a fuzzy subset of $G$. Then $\mu$ is a T-fuzzy subgroup (or simply a fuzzy subgroup) of $G$ if for all $x, y \in G$ the following properties hold

a) $\mu(e)=1$

b) $\mu(x)=\mu\left(x^{-1}\right)$

c) $T(\mu(x), \mu(y)) \leq \mu(x \circ y)$. (Closeness)

A fuzzy subset of $G$ satisfying a) and c) is called a T-fuzzy monoid or fuzzy monoid.

Proposition 2.2. Let $(G, \circ)$ be a group, e its identity element, $\mu$ a fuzzy subgroup of $G$ with $\mu(e)=1$ and $\lambda \in[0,1]$ satisfying $T(\lambda, \lambda)=\lambda$. Then the $\lambda$-cut of $\mu$ is a (crisp) subgroup of $G$. In particular, the core $H$ of $\mu$ (i.e.: the set of elements $x$ of $G$ such that $\mu(x)=1)$ is a (crisp) subgroup of $G$.

Definition 2.3. A fuzzy relation $E$ on a set $X$ is a T-indistinguishability operator (or simply indistinguishability operator) on $X$ if for all $x, y, z$ of $X$ satisfies the following properties

a) $E(x, x)=1$ (Reflexivity) 
b) $E(x, y)=E(y, x)$ (Symmetry)

c) $T(E(x, y), E(y, z)) \leq E(x, z)$ (Transitivity)

A fuzzy relation satisfying a) and c) is called a fuzzy preorder.

Indistinguishability operators extend the concept of equivalence relation and equality to the fuzzy framework and they are also called fuzzy equivalences and fuzzy equality relations. $E(x, y)$ can be viewed as the degree of similarity or indistinguishability between $x$ and $y$. A general panorama on indistinguishability operators can be found in [11].

We recall the sup $-T$ product between fuzzy relations.

Definition 2.4. Let $X, Y, Z$ be sets and $R: X \times Y \rightarrow[0,1]$ and $S: Y \times Z \rightarrow$ $[0,1]$ fuzzy relations. The sup $-T$ product $R \circ_{T} S$ of $R$ and $S$ is the fuzzy relation $R \circ_{T} S: X \times Z \rightarrow[0,1]$ defined for all $x \in X, z \in Z$ by

$$
R \circ_{T} S(x, z)=\sup _{y \in Y} T(R(x, y), S(y, z)) .
$$

To every fuzzy subset $\mu$ of a group $(G, \circ)$ a pair of fuzzy relations can be associated that are indistinguishability operators if and only if $\mu$ is a fuzzy subgroup of $G$. This two indistinguishability operators coincide when $\mu$ is a fuzzy normal subgroup and then they are compatible with the operation $\circ$ of the group.

Definition 2.5. Let $\circ$ be a binary operation on a set $G$ and $E$ a fuzzy relation on $G$. E is invariant under translations with respect to $\circ$ if for all $x, y, z \in G$,

a)

$$
E(x, y)=E(z \circ x, z \circ y) \quad(\text { left invariant })
$$

and

b)

$$
E(x, y)=E(x \circ z, y \circ z) \quad(\text { right invariant }) .
$$

Definition 2.6. Let $\mu$ be a fuzzy subset of a group $(G, \circ)$. The fuzzy relations $E_{\mu}$ and ${ }_{\mu} E$ on $G$ defined for all $x, y \in G$ by

$$
E_{\mu}(x, y)=\mu\left(x \circ y^{-1}\right)
$$


and

$$
{ }_{\mu} E(x, y)=\mu\left(y^{-1} \circ x\right)
$$

are the right and left fuzzy relations associated to $\mu$ respectively.

Proposition 2.7. Let $\mu$ be a fuzzy subgroup of a group $(G, \circ)$. Then $E_{\mu}$ and ${ }_{\mu} E$ are right and left invariant indistinguishability operators on $G$ respectively.

Lemma 2.8. If $\mu$ is a fuzzy subgroup of a group $(G, \circ)$ and $e$ is the identity element of $G$, then $E_{\mu}(x, y)=E_{\mu}\left(e, x \circ y^{-1}\right)$ and ${ }_{\mu} E(x, y)={ }_{\mu} E\left(e, y \circ x^{-1}\right)$ $\forall x, y \in G$.

Reciprocally, to every right (left) indistinguishability operator on $(G, \circ)$ a fuzzy subgroup of $G$ can be assigned.

Proposition 2.9. Let $E$ be a right (left) invariant indistinguishability operator on a group $(G, \circ)$ with identity element $e$. Then the column $\mu_{e}$ of $E$ (i.e., the fuzzy subset $\mu_{e}$ of $G$ defined by $\mu_{e}(x)=E(e, x) \forall x \in G$ ) is a fuzzy subgroup of $G$ and $E=E_{\mu_{e}}\left(E={ }_{\mu_{e}} E\right)$.

Corollary 2.10. Let $(G, \circ)$ be a group. There exist bijections between the set of fuzzy subgroups of $G$, the set of right invariant indistinguishability operators on $G$ and the set of left invariant indistinguishability operators on $G$ mapping every fuzzy subgroup $\mu$ of $G$ into its associated indistinguishability operators $E_{\mu}$ and ${ }_{\mu} E$.

The following definition fuzzifies the concept of normal subgroup.

Definition 2.11. A fuzzy subgroup $\mu$ of a group $(G, \circ)$ is a normal fuzzy subgroup if $\mu(x \circ y)=\mu(y \circ x) \forall x, y \in G$.

Proposition 2.12. Let $(G, \circ)$ be a group and $\mu$ a normal fuzzy subgroup of $G$. The indistinguishability operators $E_{\mu}$ and ${ }_{\mu} E$ associated to $\mu$ coincide and are invariant under translations.

Reciprocally,

Proposition 2.13. Let $(G, \circ)$ be a group, $\mu$ a fuzzy subgroup of $G$ and $E_{\mu}$ and ${ }_{\mu} E$ its associated indistinguishability operators. If $E_{\mu}$ and ${ }_{\mu} E$ coincide, then $\mu$ is a normal fuzzy subgroup of $G$. 
Corollary 2.14. Let $(G, \circ)$ be a group. There is a bijection between the set of normal fuzzy subgroups of $G$ and the set of indistinguishability operators on $G$ invariant under translations mapping every normal fuzzy subgroup $\mu$ of $G$ into its associated indistinguishability operators $E_{\mu}$.

In fact, the bijections of Corollaries 2.10 and 2.14 are lattice isomorphisms.

\section{Indistinguishability Operators and Fuzzy Sub- groups}

This section will deepen on the relation between fuzzy subgroups and left and right indistinguishability operators. It starts showing two ways of obtaining a fuzzy subgroup from a given fuzzy monoid (or fuzzy semi-group) $\nu$ by symmetrization and relating the associated fuzzy preorders of $\nu$ with the indistinguishability operators associated to the obtained fuzzy subgroup.

Some ways to obtain new fuzzy subgroups from a given fuzzy subgroup $\mu$ of a group $G$ are provided and at the end of the section some ways to derive fuzzy subgroups on some subgroups of the group of bijections of $G$ are shown.

Normality of a fuzzy subgroup is important because its associated indistinguishability operator and the operation of the group are compatible. In this section we provide characterizations of normal fuzzy subsets and consequently to indistinguishability operators invariant under translations.

Lemma 3.1. If $\nu$ is a fuzzy monoid of a group $(G, \circ)$, then the fuzzy subset $\nu^{\prime}$ of $G$ defined for all $x \in G$ by $\nu^{\prime}(x)=\nu\left(x^{-1}\right)$ is a fuzzy monoid.

Proof. Trivial.

In fact, considering the dual group $(G, *)$ of $(G, \circ)$ (where $*$ is defined for all $x, y \in G$ by $x * y=y \circ x)$ the mapping $f:(G, \circ) \rightarrow(G, *)$ is a group isomorphism and $\nu$ and $\nu^{\prime}$ can be considered as dual monoids.

Proposition 3.2. Let $(G, \circ)$ be a group and $\nu$ a fuzzy monoid of $G$. Then the fuzzy subset $\mu$ of $G$ defined for all $x \in G$ by

$$
\mu(x)=T\left(\nu(x), \nu\left(x^{-1}\right)\right)=T\left(\nu(x), \nu^{\prime}(x)\right)
$$

is a fuzzy subgroup of $G$. 
Proof. Properties a) and b) of Definition 2.1 are clear.

Le us prove c) i.e.: for $x, y \in G, T(\mu(x), \mu(y)) \leq \mu(x \circ y)$ :

$$
\begin{aligned}
\mu(x \circ y) & =T\left(\nu(x \circ y), \nu\left(y^{-1} \circ x^{-1}\right)\right) \\
& \geq T\left(T(\nu(x), \nu(y)), T\left(\nu\left(y^{-1}\right), \nu\left(x^{-1}\right)\right)\right) \\
& \geq T\left(T\left(\nu(x), \nu\left(x^{-1}\right), T\left(\nu(y), \nu\left(y^{-1}\right)\right)\right)\right. \\
& =T(\mu(x), \mu(y)) .
\end{aligned}
$$

Example 3.3. In $\mathbb{R}$ consider the fuzzy subset $\nu$ defined by

$$
\nu(x)= \begin{cases}1 & \text { if } x \geq 0 \\ 1+x & \text { if }-1<x<0 \\ 0 & \text { if } x \leq-1\end{cases}
$$

$\nu$ is a fuzzy monoid of $(\mathbb{R},+)$ with respect to the t-norm $E$ of Lukasiewicz and $\mu(x)=E\left(\nu(x), \nu^{\prime}(x)\right)$ is the L-fuzzy subgroup defined by

$$
\mu(x)= \begin{cases}1-|x| & \text { if }-1 \leq x \leq 1 \\ 0 & \text { otherwise. }\end{cases}
$$

In a similar way we could prove the following proposition.

Proposition 3.4. Let $(G, \circ)$ be a group and $\nu$ a fuzzy monoid of $G$. Then the fuzzy subset $\mu$ of $G$ defined for all $x \in G$ by

$$
\mu(x)=\min \left(\nu(x), \nu\left(x^{-1}\right)\right)=\min \left(\nu(x), \nu^{\prime}(x)\right)
$$

is a fuzzy subgroup of $G$.

In the same way as a fuzzy subgroup generates a left and a right invariant indistinguishability operator, a fuzzy monoid generates a right and a left invariant fuzzy preorder. The next two results show the relation between the preorders generated by $\nu$ and the indistinguishability operators of the obtained fuzzy $\mu$ in Propositions 3.2 and 3.4. 
Proposition 3.5. Let $(G, \circ)$ be a group, $\nu$ a fuzzy monoid of $G$ and $\mu$ the fuzzy subgroup of $G$ defined by $\mu(x)=T\left(\nu(x), \nu\left(x^{-1}\right)\right)=T\left(\nu(x), \nu^{\prime}(x)\right)$ for all $x \in G$. If $P$ is the right (left) invariant fuzzy preorder associated to $\nu$, then the right (left) indistinguishability operator $E$ associated to $\mu$ is $E(x, y)=$ $T(P(x, y), P(y, x))$ for all $x, y \in G$.

Proof. Let $P$ be right invariant. Then

$$
\begin{aligned}
E(x, y) & =\mu\left(x \circ y^{-1}\right) \\
& =T\left(\nu\left(x \circ y^{-1}\right), \nu\left(y \circ x^{-1}\right)\right. \\
& =T(P(x, y), P(y, x))
\end{aligned}
$$

and $E$ is also right invariant.

For left invariance a similar proof applies.

In a similar way we could prove the following result.

Proposition 3.6. Let $(G, \circ)$ be a group, $\nu$ a fuzzy monoid of $G$ and $\mu$ the fuzzy subgroup of $G$ defined by $\mu(x)=\min \left(\nu(x), \nu\left(x^{-1}\right)\right)=\min \left(\nu(x), \nu^{\prime}(x)\right)$ for all $x \in G$. If $P$ is the right (left) invariant fuzzy preorder associated to $\nu$, then the right (left) indistinguishability operator $E$ associated to $\mu$ is $E(x, y)=\min (P(x, y), P(y, x))$ for all $x, y \in G$.

There are several ways to obtain a fuzzy subgroup from a given one. Some interesting ones are Propositions 3.7, 3.9 and 3.10.

Proposition 3.7. Let $\mu$ be a T-fuzzy subgroup of a group $(G, \circ), T$ a continuous Archimedean t-norm with an additive generator $t$, and $f$ a map $f:[0,1] \rightarrow[0,1]$. If there exists a metric transform $s$ (i.e.: a sub-additive and non-decreasing map $s:[0, \infty) \rightarrow[0, \infty)$ with $s(0)=0)$ such that $f=t^{[-1]} \circ s \circ t$, then $f \circ \mu$ is a $T$-fuzzy subgroup of $G$.

Proof.

a) $f(\mu(e))=\left(t^{[-1]} \circ s \circ t\right)(\mu(e))=1$.

b) It is clear that $f(\mu(x))=f\left(\mu\left(x^{-1}\right)\right)$

c)

$$
\left.\left.T(f(\mu(x)), f(\mu(y)))=t^{[-1]}\left(t\left(t^{[-1]} \circ s \circ t\right)(\mu(x))\right)+t\left(t^{[-1]} \circ s \circ t\right)(\mu(y))\right)\right) .
$$


i) If $(s \circ t)(\mu(x))>t(0)$ or $(s \circ t)(\mu(y))>t(0)$, then $\left(t^{[-1]} \circ s \circ t\right)(\mu(x))=$ 0 or $\left(t^{[-1]} \circ s \circ t\right)(\mu(y))=0$ and therefore $T(f(\mu(x)), f(\mu(y))) \leq$ $f(\mu(x \circ y))$.

ii) Otherwise, $t(\mu(x))+t(\mu(y)) \geq t(\mu(x \circ y))$ and

$$
\begin{aligned}
T(f(\mu(x)), f(\mu(y))) & =t^{-1}((t \circ f)(\mu(x))+(t \circ f)(\mu(y))) \\
& =t^{-1}\left(\left(t \circ t^{-1} \circ s \circ t\right)(\mu(x))+\left(t \circ t^{-1} \circ s \circ t\right)(\mu(y))\right) \\
& =t^{-1}((s \circ t)(\mu(x))+(s \circ t)(\mu(y))) \\
& \leq\left(t^{-1} \circ s \circ t\right)(\mu(x \circ y)) \\
& =f(\mu(x \circ y)) .
\end{aligned}
$$

Example 3.8. The map $s:[0, \infty) \rightarrow[0, \infty)$ defined by $s(x)=x^{\alpha}$ for all $x \in[0, \infty)$ with $0<\alpha \leq 1$ is a metric transform. Consider a group $(G, \circ)$.

a) If $\mu$ is a $T$-fuzzy subgroup of $G$ with $T$ the Eukasiewicz $t$-norm, $t(x)=$ $1-x$ an additive generator of $T$, then

$$
f(\mu(x))=\left(t^{-1} \circ s \circ t\right)(\mu(x))=1-(1-\mu(x))^{\alpha}
$$

is also a $T$-fuzzy subgroup of $G$.

b) If $\mu$ is a $T$-fuzzy subgroup of $G$ with $T$ the Product $t$-norm and $t(x)=$ $-\ln x$ an additive generator of $T$, then

$$
f(\mu(x))=e^{-(-\ln \mu(x))^{\alpha}}
$$

is also a $T$-fuzzy subgroup of $G$.

Proposition 3.9. Let $\mu$ be a min-fuzzy subgroup of a group $(G, \circ)$ and $f$ : $[0,1] \rightarrow[0,1]$ a non-decreasing map with $f(1)=1$. Then $f \circ \mu$ is a min-fuzzy subgroup of $G$.

Proof. Trivial.

If $E$ is an indistinguishability operator on a set $X$ and $f: X \rightarrow X$ is a map, then the fuzzy relation $E_{f}$ on $X$ defined for all $x, y \in X$ by $E_{f}(x, y)=E(f(x), f(y))$ clearly is an indistinguishability operator on $X$. Hence, mappings $f$ preserving invariance under translations will provide a way to obtain new fuzzy subgroups from a given one. 
Proposition 3.10. Let $\mu$ be a fuzzy subgroup of $(G, \circ), E_{\mu}$ its right invariant associated indistinguishability operator and $f: G \rightarrow G$ a bijection. If $\left(E_{\mu}\right)_{f}$ is right invariant, then its associated fuzzy subgroup $\mu_{f}$ is $\mu_{f}(x)=\mu(f(x) \circ$ $\left.(f(e))^{-1}\right)$.

Proof.

$$
\begin{aligned}
\mu_{f}(x) & =\left(E_{\mu}\right)_{f}(x, e) \\
& =E_{\mu}(f(x), f(e)) \\
& =E_{\mu}\left(f(x) \circ(f(e))^{-1}, e\right) \\
& =\mu\left(f(x) \circ(f(e))^{-1}\right) .
\end{aligned}
$$

Similarly,

Proposition 3.11. Let $\mu$ be a fuzzy subgroup of $(G, \circ),{ }_{\mu} E$ its left invariant associated indistinguishability operator and $f: G \rightarrow G$ a bijection. If $\left({ }_{\mu} E\right)_{f}$ is left invariant, then its associated fuzzy subgroup ${ }_{f} \mu$ is ${ }_{f} \mu(x)=\mu\left((f(e))^{-1}\right.$ o $f(x))$.

Example 3.12. Let $(G, \circ)$ be a group and $\mu$ a fuzzy subgroup of $G$.

a) The fuzzy subset $\nu$ of $G$ defined for all $x \in G$ by $\nu(x)=\mu\left(x^{-1}\right)$ is a fuzzy subgroup of $X$ and $E_{\nu}(x, y)=E_{\mu}\left(x^{-1}, y^{-1}\right)$.

The following common actions of $G$ on $G$ also provide new fuzzy subgroups.

b) Fixing $z \in G$, from the conjugation $f_{z}(x)=z \circ x \circ z^{-1}$ we obtain $E_{f_{z}}(x, y)=E\left(z \circ x \circ z^{-1}, z \circ y \circ z^{-1}\right)$ and $\mu_{f_{z}}(x)=\mu\left(z \circ x \circ z^{-1}\right)$.

c) Fixing $z \in G$, from the right translation $f_{z}(x)=x \circ z$ we obtain $E_{f_{z}}(x, y)=E(x \circ z, y \circ z)$. If $E$ is left invariant, then $E_{f_{z}}(x, y)=$ $E(x \circ z, y \circ z)=E\left(z^{-1} \circ x \circ z, z^{-1} \circ y \circ z\right)$ and $\mu_{f_{z}}(x)=\mu\left(z^{-1} \circ x \circ z\right)$.

c) Fixing $z \in G$, from the left translation ${ }_{z} f(x)=z \circ x$ we obtain $E_{z}(x, y)=$ $E(z \circ x, z \circ y)$. If $E$ is right invariant, then $E_{z}(x, y)=E(z \circ x, z \circ y)=$ $E\left(z \circ x \circ z^{-1}, z \circ y \circ z^{-1}\right)$ and $\mu_{z}(x)=\mu\left(z \circ x \circ z^{-1}\right)$.

Normality is an important property of fuzzy subgroups because their associated indistinguishability operators are invariant under translations and the operation is compatible with them. The next propositions provide characterizations for normal fuzzy subgroups. 
Proposition 3.13. A fuzzy subgroup $\mu$ of $(G, \circ)$ is normal if and only if for all $x, y, z, t \in G, \mu\left(x \circ y \circ(z \circ t)^{-1}\right) \geq T\left(\mu\left(x \circ z^{-1}\right), \mu\left(y \circ t^{-1}\right)\right)$.

Proof.

$\Rightarrow$ ) If $\mu$ is normal, then

$$
\begin{aligned}
\mu\left(x \circ y \circ t^{-1} \circ z^{-1}\right) & =\mu\left(z^{-1} \circ x \circ y \circ t^{-1}\right) \\
& \geq T\left(\mu\left(z^{-1} \circ x\right), \mu\left(y \circ t^{-1}\right)\right) \\
& =T\left(\mu\left(x \circ z^{-1}\right), \mu\left(y \circ t^{-1}\right)\right) .
\end{aligned}
$$

$\Leftarrow)$ By symmetry, it is sufficient to prove $\mu(y \circ x) \geq \mu(x \circ y)$.

$$
\begin{aligned}
\mu(y \circ x) & =\mu\left(y \circ x \circ y \circ y^{-1}\right) \\
& \geq T\left(\mu\left(y \circ y^{-1}\right), \mu(x \circ y)\right) \\
& =\mu(x \circ y) .
\end{aligned}
$$

The result of Proposition 3.13 can be rewritten in a more convenient manner as in the next corollary.

Corollary 3.14. A fuzzy subgroup $\mu$ of $(G, \circ)$ is normal if and only if for all $x, y, z, t \in G, \mu(x \circ z \circ t \circ y) \geq T(\mu(x \circ y), \mu(z \circ t))$.

Proposition 3.13 has the following translation to indistinguishability operators.

Proposition 3.15. An indistinguishability operator $E$ on a group $(G, \circ)$ is invariant under translations if and only if for all $x, y, z, t \in G E(x \circ y, z \circ t) \geq$ $T(E(x, z), E(y, t))$.

Proof. $E$ is the indistinguishability operator associated to a normal fuzzy subgroup $\mu$ of $G$. Then

$$
\begin{aligned}
E(x \circ y, z \circ t) & =\mu\left(x \circ y \circ(z \circ t)^{-1}\right) \\
& \geq T\left(\mu\left(x \circ z^{-1}\right), \mu\left(y \circ t^{-1}\right)\right) \\
& =T(E(x, z), E(y, t)) .
\end{aligned}
$$


Proposition 3.16. [8] A fuzzy subgroup $\mu$ of $(G, \circ)$ is normal if and only if for all $x, y \in G, \mu(x)=\mu\left(y \circ x \circ y^{-1}\right)$.

The next proposition is the counterpart of this last result to indistinguishability operators.

Proposition 3.17. Let $E$ be an indistinguishability operator on a group $(G, \circ)$.

a) If $E$ is invariant under translations, then for all $x, y \in G E(x, y)=$ $E\left(x^{-1}, y^{-1}\right)$.

b) If $E(x, y)=E\left(x^{-1}, y^{-1}\right)$ for all $x, y \in G$ and $E$ is left (right) invariant, then $E$ is invariant under translations.

Proof.

a)

$$
\begin{array}{r}
E(x, y)=E\left(x \circ x^{-1}, y \circ x^{-1}\right)=E\left(e, y \circ x^{-1}\right)=\mu\left(y \circ x^{-1}\right) \\
E\left(x^{-1}, y^{-1}\right)=E\left(x^{-1} \circ x, y^{-1} \circ x\right)=E\left(e, y^{-1} \circ x\right)=\mu\left(y^{-1} \circ x\right) .
\end{array}
$$

But $\mu\left(y \circ x^{-1}\right)=\mu\left(y^{-1} \circ x\right)$ because $E$ is invariant under translations and, hence, $\mu$ is normal.

b) If $E$ is left invariant, then there exists a fuzzy subgroup of $G$ with $E(x, y)=\mu\left(x^{-1} \circ y\right)$ and $E\left(x^{-1}, y^{-1}\right)=\mu\left(x \circ y^{-1}\right)=\mu\left(y \circ x^{-1}\right)$. So $\mu$ is normal and hence $E$ is invariant under translations.

A similar proof applies if $E$ is right invariant.

The next proposition shows a way to obtain a right invariant indistinguishability operator $E_{r}$ from an arbitrary indistinguishability operator $E$ and therefore a way to obtain fuzzy subgroups. Moreover, if $E$ is left invariant, then the obtained fuzzy subgroup is normal.

Proposition 3.18. Let $E$ be an indistinguishability operator on a group $(G, \circ)$. Consider the indistinguishability operator $E_{r}$ on $G$ and the fuzzy subset of $G$ defined for all $x, y \in G$ by

$$
E_{r}(x, y)=\inf _{z \in G} E(x \circ z, y \circ z)
$$




$$
\mu_{r}(x)=\inf _{z \in G} E(x \circ z, z)
$$

Then

a) $E_{r}$ is right invariant and $\mu_{r}$ is a fuzzy subgroup.

b) If $E$ is left invariant, then $E_{r}$ is invariant under translations and $\mu_{r}$ is normal.

c) If $E$ is right invariant, then $E_{r}=E$.

Proof.

a)

$$
\begin{gathered}
E_{r}(x \circ t, y \circ t)=\inf _{z \in G} E(x \circ t \circ z, y \circ t \circ z)=\inf _{u \in G} E(x \circ u, y \circ u)=E_{r}(x, y) . \\
\mu(x)=\inf _{z \in G} E(x \circ z, z)=E_{r}(x, e) .
\end{gathered}
$$

b)

$$
E_{r}(t \circ x, t \circ y)=\inf _{z \in G} E(t \circ x \circ z, t \circ y \circ z)=\inf _{z \in G} E(x \circ z, y \circ z)=E_{r}(x, y) .
$$

So $E_{r}$ is left invariant and thanks to a) it is also right invariant.

c) If $E$ is right invariant, then

$$
E_{r}(x, y)=\inf _{z \in G} E(x \circ z, y \circ z)=E(x, y)
$$

The next proposition is dual to Proposition 3.18.

Proposition 3.19. Let $E$ be an indistinguishability operator on a group $(G, \circ)$. Consider the indistinguishability operator $E_{l}$ on $G$ and the fuzzy subset of $G$ defined for all $x, y \in G$ by

$$
\begin{aligned}
E_{l}(x, y) & =\inf _{z \in G} E(z \circ x, z \circ y), \\
\mu_{l}(x) & =\inf _{z \in G} E(z \circ x, z)
\end{aligned}
$$

Then 
a) $E_{l}$ is left invariant and $\mu_{l}$ is a fuzzy subgroup.

b) If $E$ is right invariant, then $E_{l}$ is invariant under translations and $\mu_{l}$ is normal.

c) If $E$ is left invariant, then $E_{l}=E$.

From an indistinguishability operator $E$ on a group $(G, \circ)$, several indistinguishability operators on the set of mappings $f: G \rightarrow G$ can be obtained. Considering their restrictions to the group $B$ (under composition) of bijective mappings $f: G \rightarrow G$, if $E$ is right (left) invariant, then the obtained indistinguishability operators are right (left) invariant and hence from fuzzy subgroups of $G$ fuzzy subgroups of $B$ can be obtained.

Proposition 3.20. [11] Let $E$ be an indistinguishability operator on a set $X, x \in X$ and $F$ the set of mappings $f: X \rightarrow X$. Then the fuzzy relation $E_{x}$ on $F$ defined for all $f, g \in F$ by $E_{x}(f, g)=E(f(x), g(x))$ is an indistinguishability operator.

Corollary 3.21. [11] Let $E$ be an indistinguishability operator on a set $X$ and $F$ the set of mappings $f: X \rightarrow X$. Then the fuzzy relation $E_{F}$ on $F$ defined for all $f, g \in F$ by $E_{F}(f, g)=\inf _{x \in X} E(f(x), g(x))$ is an indistinguishability operator.

If $X$ is a group, we get the following result concerning invariance.

Proposition 3.22. Let $E$ be an indistinguishability operator on a group $(G, \circ)$ and $B$ the group (under composition) of bijective mappings $f: G \rightarrow G$. Then $E_{B}\left(E_{F}\right.$ restricted to $\left.B\right)$ is a right invariant indistinguishability operator.

Proof. We only need to prove that $E_{B}$ is right invariant under translations.

$$
\begin{aligned}
E_{B}(f \circ h, g \circ h) & =\inf _{x \in G} E(f(h(x)), g(h(x))) \\
& =\inf _{y \in G} E(f(y), g(y)) \\
& =E_{B}(f, g) .
\end{aligned}
$$

Corollary 3.23. If $\mu$ is a fuzzy subgroup of $(G, \circ)$. Then the fuzzy subset $\mu_{B}$ of $B$ defined for all $f \in B$ by $\mu_{B}(f)=\inf _{x \in G} \mu\left(f(x) \circ x^{-1}\right)$ is a fuzzy subgroup of $B$. 
Proof. Let $E_{\mu}$ be the right invariant indistinguishability operator associated to $\mu$. Then $\left(E_{\mu}\right)_{B}$ is a right invariant indistinguishability operator on $B$ and its associated fuzzy subgroup $\mu$ is defined for all $f \in B$ by $\mu(f)=$ $\left(E_{\mu}\right)_{B}(f, i d)=\inf _{x \in G} E_{\mu}(f(x), x)=\inf _{x \in G} \mu\left(f(x) \circ x^{-1}\right)=\mu_{B}(f)$.

\section{Relationship between Natural, Right and Left Invariant Indistinguishability Opera- tors}

A fuzzy subgroup $\mu$ of a group $G$ is in particular a fuzzy subset of $G$ and therefore it generates an indistinguishability operator $E^{\mu}$ in a natural way (Definition 4.3). In this section the relation between this operator and the left and right ones associated to $\mu\left(E_{\mu}\right.$ and $\left.{ }_{\mu} E\right)$ will be studied. In particular it will be proved that $E^{\mu} \geq \max \left(E_{\mu}, \mu E\right)$. In general equality is not given; in fact, in order to achieve equality $\mu$ must be normal and $G$ must be idempotent.

In this section the t-norm $T$ will be assumed to be left continuous.

Definition 4.1. $[1,9]$

- The residuation $\vec{T}$ of a $t$-norm $T$ is the $\operatorname{map} \vec{T}:[0,1] \times[0,1] \rightarrow[0,1]$ defined for all $x, y \in[0,1]$ by

$$
\vec{T}(x \mid y)=\sup \{\alpha \in[0,1] \mid T(x, \alpha) \leq y\}
$$

- The biresiduation $\stackrel{\leftrightarrow}{T}$ of a t-norm $T$ is the $\operatorname{map} \overleftrightarrow{T}:[0,1] \times[0,1] \rightarrow[0,1]$ defined for all $x, y \in[0,1]$ by

$$
\overleftrightarrow{T}(x \mid y)=\min \{\vec{T}(x \mid y), \vec{T}(y \mid x)\}
$$

If $T$ is used to model the logical conjunction, then the residuation and biresiduation model the implication and biimplication respectively.

Proposition 4.2. $[14,11] \stackrel{\leftrightarrow}{T}$ is an indistinguishability operator on $[0,1]$ and is also denoted by $E_{T}$ and called the natural indistinguishability operator associated to $T$. 
Proposition 4.3. [14] If $\mu$ is a fuzzy subset of a set $X$, then $E^{\mu}$ defined by $E^{\mu}(x, y)=E_{T}(\mu(x), \mu(y))$ for all $x, y \in X$ is an indistinguishability operator on $X$.

Lemma 4.4. If $\mu$ is a fuzzy subgroup of the group $(G, \circ)$, then $E^{\mu}(x, y)=$ $E^{\mu}\left(x, y^{-1}\right)=E^{\mu}\left(x^{-1}, y^{-1}\right)$ for all $x, y \in G$.

Proof.

$$
E^{\mu}(x, y)=E_{T}(\mu(x), \mu(y))=E_{T}\left(\mu(x), \mu\left(y^{-1}\right)\right)=E_{T}\left(\mu\left(x^{-1}\right), \mu\left(y^{-1}\right)\right) .
$$

Corollary 4.5. If $\mu$ is a fuzzy subgroup of a group $(G, \circ)$, then $E^{\mu}\left(x, x^{-1}\right)=$ 1 for all $x \in G$.

Proof.

$$
E^{\mu}\left(x, x^{-1}\right)=E^{\mu}(x, x)=1 .
$$

Proposition 4.6. Let $\mu$ be a fuzzy subgroup of of a group $(G, \circ)$. Then $E^{\mu}(x, y) \geq \mu(x \circ y)$ for all $x, y \in G$.

Proof.

$$
T(\mu(x \circ y), \mu(x))=T\left(\mu(x \circ y), \mu\left(x^{-1}\right)\right) \leq \mu(y)
$$

which is equivalent to

$$
\vec{T}(\mu(x) \mid \mu(y)) \geq \mu(x \circ y) .
$$

In a similar way, $\vec{T}(\mu(y) \mid \mu(x)) \geq \mu(x \circ y)$.

As a corollary we have the following proposition.

Proposition 4.7. Let $\mu$ be a fuzzy subgroup of $G$. Then $E^{\mu}(x, y) \geq E_{\mu}(x, y)$ and $E^{\mu}(x, y) \geq_{\mu} E(x, y)$ for all $x, y \in G$, where $E_{\mu}(x, y)=\mu\left(x \circ y^{-1}\right)$ and ${ }_{\mu} E(x, y)=\mu\left(x^{-1} \circ y\right)$.

Proof.

$$
\begin{aligned}
& E^{\mu}(x, y)=E^{\mu}\left(x, y^{-1}\right) \geq \mu\left(x \circ y^{-1}\right)=E_{\mu}(x, y) \\
& E^{\mu}(x, y)=E^{\mu}\left(x^{-1}, y\right) \geq \mu\left(x^{-1} \circ y\right)={ }_{\mu} E(x, y)
\end{aligned}
$$


As an alternative proof, we can see that $\mu$, being a column of $E_{\mu}$ and of ${ }_{\mu} E$, is extensional with respect to these two indistinguishability operators, which is equivalent to the assertion of the proposition.

Proposition 4.8. Let $\mu$ be a fuzzy subgroup of $G$ and $x, y, z \in G$. Then $E^{\mu}(z \circ x, z \circ y) \geq_{\mu} E(x, y)$ and $E^{\mu}(x \circ z, y \circ z) \geq_{\mu} E(x, y)$.

Proof.

$$
\begin{aligned}
E^{\mu}(z \circ x, z \circ y) & =E_{T}(\mu(z \circ x), \mu(z \circ y)) \\
& =E_{T}\left(\mu E\left(z^{-1}, x\right),{ }_{\mu} E\left(z^{-1}, y\right)\right) \geq_{\mu} E(x, y) . \\
E^{\mu}(x \circ z, y \circ z) & =E_{T}(\mu(x \circ z), \mu(y \circ z)) \\
& =E_{T}\left(E_{\mu}\left(x, z^{-1}\right), E_{\mu}\left(y, z^{-1}\right)\right) \geq E_{\mu}(x, y) .
\end{aligned}
$$

Proposition 4.9. Let $\mu$ be a fuzzy subgroup of $G$. If $E^{\mu}$ is invariant under translations, then $E^{\mu}=E_{\mu}={ }_{\mu} E$ and $\mu$ is a normal fuzzy subgroup.

Proof. If $E^{\mu}$ is invariant under translations, then there exists a normal fuzzy subgroup $\nu$ of $G$ such that $E^{\mu}=E_{\nu}$. For $x \in G$,

$$
\mu(x)=E_{T}(\mu(x), \mu(e))=E_{\nu}(x, e)=\nu\left(x \circ e^{-1}\right)=\nu(x) .
$$

Corollary 4.10. If $E^{\mu}$ is invariant under translations, then for all $x \in G$, $\mu\left(x^{2}\right)=1$.

Proof.

$$
1=E^{\mu}\left(x, x^{-1}\right)=E_{\mu}\left(x, x^{-1}\right)=\mu(x \circ x) .
$$

This result shows the difficulty for $E^{\mu}$ to be invariant under translations. This will be analyzed in the examples of the section of Concluding Remarks.

Definition 4.11. [12] Two fuzzy relations $E$ and $F$ on a universe $X$ commute or are permutable if $R \circ S=S \circ R$. 
Proposition 4.12. [4] Let $E$ and $F$ be two indistinguishability operators on a universe $X$. Then $E$ and $F$ are permutable if and only if $E \circ F$ is an indistinguishability operator. Moreover, this occurs if and only if $E \circ F$ coincides with the transitive closure $\overline{\max (E, F)}$ of $\max (E, F)$.

Proposition 4.13. [12] If $E_{\mu}$ and ${ }_{\nu} E$ are the right and left invariant indistinguishability operators associated to two fuzzy subgroups $\mu$ and $\nu$ of a group $(G, \circ)$ respectively, then they commute. In particular, $\max \left(E_{\mu}, E\right)$ is an indistinguishability operator.

Corollary 4.14. If $\mu$ is a fuzzy subgroup of a group $(G, \circ)$, then $E^{\mu} \geq$ $\max \left(E_{\mu, \mu} E\right)$.

\section{Double cosets}

For two subgroups $H$ and $K$ of a group $G$, a double coset is an equivalence class of the equivalence relation for which two elements of $G$ are equivalent if they differ by left multiplication with an element in $H$ and right multiplication with an element of $K$. In this way the interrelation between the left equivalence relation associated to $H$ and the right equivalence relation associated to $K$ can be studied.

This section fuzzifies the concept of double coset on a group $G$.

Definition 5.1. Let $(G, \circ)$ be a group and $\mu, \nu$ fuzzy subgroups of $G$. A fuzzy subset $D$ of $G$ is a fuzzy double coset for $\mu$ and $\nu$ if there exists $x \in G$ such that

$$
D(y)=\sup _{k \in G} T\left(\nu(k), \mu\left(x \circ k \circ y^{-1}\right)\right)=\sup _{h \in G} T\left(\mu(h), \nu\left(x^{-1} \circ h \circ y\right)\right)
$$

for all $y \in G$. We will denote $D$ also by $D_{x}$ or $D_{x}^{\mu, \nu}$.

Proposition 5.2. The fuzzy relation $E_{\mu, \nu}$ on $G$ defined by $E_{\mu, \nu}(x, y)=D_{x}(y)$ for all $x, y \in G$ is an indistinguishability operator.

Proof.

Reflexivity

$$
E_{\mu, \nu}(x, x)=\sup _{k \in G} T\left(\nu(k), \mu\left(x \circ k \circ x^{-1}\right)\right) \geq T(\nu(e), \mu(e))=1 .
$$




\section{Symmetry}

$$
\begin{aligned}
E_{\mu, \nu}(x, y) & =\sup _{k \in G} T\left(\nu(k), \mu\left(x \circ k \circ y^{-1}\right)\right) \\
& =\sup _{k \in G} T\left(\nu\left(k^{-1}\right), \mu\left(y \circ k^{-1} \circ x^{-1}\right)\right)=E_{\mu, \nu}(y, x) .
\end{aligned}
$$

Transitivity

$$
\begin{aligned}
T\left(E_{\mu, \nu}(x, y), E_{\mu, \nu}(y, z)\right) & =T\left(\sup _{k \in G} T\left(\nu(k), \mu\left(x \circ k \circ y^{-1}\right)\right), \sup _{l \in G} T\left(\nu(l), \mu\left(y \circ l \circ z^{-1}\right)\right)\right) \\
& =\sup _{k, l \in G} T\left(\nu(k), \nu(l), \mu\left(x \circ k \circ y^{-1}\right), \mu\left(y \circ l \circ z^{-1}\right)\right. \\
& \leq \sup _{k, l \in G} T\left(\nu(k \circ l), \mu\left(x \circ k \circ y^{-1} \circ y \circ l \circ z^{-1}\right)\right) \\
& =\sup _{k, l \in G} T\left(\nu(k \circ l), \mu\left(x \circ k \circ l \circ z^{-1}\right)\right)=E_{\mu, \nu}(x, z) .
\end{aligned}
$$

The duality between $D_{x}^{\mu, \nu}$ and $D_{x}^{\nu, \mu}$ is stated in the following proposition.

Proposition 5.3. Let $G$ be a group and $\mu, \nu$ fuzzy subgroups of $G$. For all $x, y \in G$ we have $E_{\mu, \nu}(x, y)=E_{\nu, \mu}\left(x^{-1}, y^{-1}\right)$.

Proof.

$$
\begin{aligned}
E_{\mu, \nu}(x, y) & =D_{x}^{\mu, \nu}(y) \\
& =\sup _{k \in G} T\left(\nu(k), \mu\left(x \circ k \circ y^{-1}\right)\right) \\
& =\sup _{h \in G} T\left(\mu(h), \nu\left(x^{-1} \circ h \circ y\right)\right) \\
& =D_{x^{-1}}^{\nu, \mu}\left(y^{-1}\right) \\
& =E_{\nu, \mu}\left(x^{-1}, y^{-1}\right) .
\end{aligned}
$$

Special case when $\mu$ or $\nu$ are the trivial subgroup $\{e\}$ :

\section{Proposition 5.4.}

a) If $\nu=\{e\}$, then the fuzzy double cosets of $\mu$ and $\nu$ are the fuzzy right cosets of $\mu$. 
Proof. a)

$$
\begin{aligned}
D_{x}^{\mu,\{e\}}(y) & =\sup _{k \in G} T\left(\{e\}(k), \mu\left(x \circ k \circ y^{-1}\right)\right) \\
& =T\left(\{e\}(e), \mu\left(x \circ e \circ y^{-1}\right)\right) \\
& =\mu\left(x \circ y^{-1}\right) .
\end{aligned}
$$

b)

$$
\begin{aligned}
D_{x}^{\{e\}, \nu}(y) & =\sup _{h \in G} T\left(\{e\}(h), \nu\left(x^{-1} \circ h \circ y\right)\right) \\
& =T\left(\{e\}(e), \nu\left(x^{-1} \circ e \circ y\right)\right) \\
& =\mu\left(x^{-1} \circ y\right) .
\end{aligned}
$$

Proposition 5.5. Let $\mu$ be a normal fuzzy subgroup of $G$. Then the double cosets for $\mu$ and $\mu$ coincide with the right and left fuzzy cosets of $\mu$.

Proof.

$$
\begin{aligned}
D_{x}^{\mu, \mu}(y) & =\sup _{k \in G} T\left(\mu(k), \mu\left(x \circ k \circ y^{-1}\right)\right. \\
& =\sup _{k \in G} T\left(\mu(k), \mu\left(y^{-1} \circ x \circ k\right)\right. \\
& \geq T\left(\mu(e), \mu\left(y^{-1} \circ x \circ e\right)\right)=\mu\left(y^{-1} \circ x\right)
\end{aligned}
$$

because $\mu$ is normal.

But

$$
\sup _{k \in G} T\left(\mu(k), \mu\left(y^{-1} \circ x \circ k\right)=\sup _{k \in G} T\left(\mu\left(k^{-1}\right), \mu\left(y^{-1} \circ x \circ k\right) \leq \mu\left(y^{-1} \circ x\right)\right.\right.
$$

because $\mu$ is a fuzzy subgroup. 


\section{Indistinguishability Operators as Fuzzy Sub- groups}

If $(G, \circ)$ is a group, then $G \times G$ is also a group. Being a fuzzy relation on $G$ a fuzzy subset of $G \times G$, it becomes a candidate to be a fuzzy subgroup of $G \times G$. This question is studied in this section.

The next lemma is a re-writing of Proposition 3.15.

Lemma 6.1. Let $E$ be an indistinguishability operator on a group $(G, \circ)$. $E$ is closed under products in $G \times G$ if and only if $E$ is invariant under translations.

Proposition 6.2. Let $E$ be an indistinguishability operator on a group $G$. The following are equivalent:

i) $E$ is closed under products in $G \times G$.

ii) $E$ is a fuzzy subgroup of $G \times G$.

Proof.

i) $\Rightarrow$ ii)

$$
\begin{aligned}
E(x, y) & =T\left(E\left(x^{-1}, x^{-1}\right), E(x, y)\right) \leq E\left(e, x^{-1} \circ y\right) \\
& =T\left(E\left(e, x^{-1} \circ y\right), E\left(y^{-1}, y^{-1}\right)\right) \\
& \leq E\left(y^{-1}, x^{-1}\right)=E\left(x^{-1}, y^{-1}\right)
\end{aligned}
$$

and equality follows from symmetry.

ii) $\Rightarrow$ i) is trivial.

Proposition 6.3. Let $E$ be an indistinguishability operator on a group $G$. If $E$ is a fuzzy subgroup of $G \times G$, then the fuzzy subset $\mu$ of $G$ defined for all $x \in G$ by $\mu(x)=E(e, x)$ (i.e., the column $\mu_{e}$ of $E$ ) is a normal fuzzy subgroup of $G$.

Proof. It is a consequence of Lemma 6.1.

Reciprocally, 
Proposition 6.4. Let $\mu$ be a normal fuzzy subgroup of a group $G$. The fuzzy relation $E_{\mu}$ defined for all $x, y \in G$ by $E_{\mu}(x, y)=\mu\left(x \circ y^{-1}\right)$ is a fuzzy subgroup of $G \times G$.

Proof. $E_{\mu}$ is an indistinguishability operator invariant under translations. So, from Lemma 6.1 it is closed under products and thanks to Proposition 6.2 it is a fuzzy subgroup of $G \times G$.

Invariance under translations for a fuzzy relation is essential in many applications. In the introductory section, for instance, its need in Fuzzy Mathematical Morphology is pointed out. Then, from an algebraic point of view, the structural element turns out to be then a (normal) fuzzy subgroup. Similarly occurs in $\left(\mathbb{R}^{3},+\right)$ when considering voxels instead of pixels. Another example can be found in music (and in signal theory), where the relation between sounds (notes) is measured by the quotient of their frequencies, so that we obtain a fuzzy equivalence relation on $\left(\mathbb{R}^{+},\right)$invariant under products. The results of this section provide an alternative way of considering the invariance; namely by stating that this property for an indistinguishability operator $E$ on $(G, \circ)$ is equivalent to being a fuzzy subgroup of $G \times G$. Thanks to this, some results of Section 3 can be rewritten in a more algebraic way. For example

Proposition. A fuzzy subset $\mu$ of a group $(G, \circ)$ is a normal fuzzy subgroup of $G$ if and only if the fuzzy subset $E$ of $G \times G$ defined for all $x, y \in G$ by $E(x, y)=\mu\left(x \circ y^{-1}\right)$ is a fuzzy subgroup of $G \times G$.

\section{Concluding Remarks}

In this paper we have studied fuzzy subgroups taking into account the close relation between them and their associated left and right invariant indistinguishability operators. In this way different ways to obtain new fuzzy subgroups from a given one are provided. The relation between the associated invariant indistinguishability operators to a fuzzy subgroup $\mu$ and the operator $E^{\mu}$ generated by $\mu$ as a fuzzy subset is also analyzed. The conditions in which a fuzzy relation $R$ on a group $G$ can be seen as a fuzzy subgroup of $G \times G$ are obtained.

There is an important relation between Propositions 2.7 and 2.9 and the results of Section 4 and 5 of [6]. In fact, these two propositions can be 
regarded as particular cases of Theorems 11 and 13 of [6]. Indeed, given a set $S$, consider the set $\Sigma_{S}$ of permutations or bijective maps of $S$ which is a group under composition. Then Theorem 11 of [6] states that if $\mu$ is a fuzzy subgroup of $\Sigma_{S}$, then the fuzzy relation $E_{\mu}$ on $S$ defined for all $x, y \in S$ by $E_{\mu}(x, y)=\sup _{g \in \Sigma_{S}}\{\mu(g) \mid g(x)=y\}$ is an indistinguishability operator. Reciprocally, Theorem 13 of [6] states that given an indistinguishability operator $E$ on $S$, the fuzzy subset $\mu$ of $\Sigma_{S}$ defined for all $f \in \Sigma_{S}$ by $\mu(f)=\inf _{x \in S} E(x, f(x))$ is a fuzzy subgroup. If $S$ is a group $(G, \cdot)$, then the mapping $\langle\cdot\rangle$ : $G \rightarrow \Sigma_{G}$ sending $x$ to $\langle x>$ defined by $\langle x\rangle(y)=x \cdot y$ is an embedding of $g$ in $\Sigma_{G}$ and to every fuzzy subgroup $\mu$ of $G$ corresponds a fuzzy subgroup $\mu^{\prime}$ of $\Sigma_{G}$ defined for all $f \in \Sigma_{G}$ by $\mu^{\prime}(f)=\mu(x)$ if $f=<x>$ and 0 otherwise. Then for all $x, y \in G, E_{\mu}(x, y)=E_{\mu^{\prime}}(x, y)=\mu\left(x \cdot y^{-1}\right)=$ $\left.\mu^{\prime}(<x>0<y\rangle^{-1}\right)$ and we recover Propositions 2.7 and 2.9.

Let us finish this work with a couple of examples on the real line and a third one on the plane.

Example 7.1. For the t-norm $T$ of Eukasiewicz, the fuzzy set $\mu$ of $(\mathbb{R},+)$ defined by all $x \in \mathbb{R}$ by $\mu(x)=\max (1-|x|, 0)$ (a triangular fuzzy number) is a fuzzy subgroup (normal because $\mathbb{R}$ is an Abelian group). Its associated indistinguishability operator $E$ is defined for all $x, y \in \mathbb{R}$ by $E(x, y)=\mu(x-$ $y)=\max (1-|x-y|, 0)$. The indistinguishability operator $E^{\mu}$ is defined by $E^{\mu}(x, y)=1-|\mu(x)-\mu(y)|$. They coincide in $[0,1]^{2}$ and in $[-1,0]^{2}$.

Example 7.2. For the Product t-norm, the fuzzy set $\mu$ of $\left(\mathbb{R}^{+}, \cdot\right)$ defined by all $x \in \mathbb{R}$ by

$$
\mu(x)= \begin{cases}x & \text { if } x \leq 1 \\ \frac{1}{x} & \text { otherwise }\end{cases}
$$

is a fuzzy subgroup (normal because $\mathbb{R}^{+}$is an Abelian group). Its associated indistinguishability operator $E$ is defined for all $x, y \in \mathbb{R}$ by

$$
E_{\mu}(x, y)=\mu\left(\frac{x}{y}\right)= \begin{cases}\frac{x}{y} & \text { if } x \leq y \\ \frac{y}{x} & \text { otherwise. }\end{cases}
$$


The indistinguishability operator $E^{\mu}$ is defined by

$$
E^{\mu}(x, y)=\min \left(\frac{\mu(x)}{\mu(y)}, \frac{\mu(y)}{\mu(x)}\right)= \begin{cases}\frac{x}{y} & \text { if } x \leq y \leq 1 \\ \frac{y}{x} & \text { if } x \geq y \geq 1 \\ x \cdot y & \text { if } x \geq 1 \text { and } x \geq \frac{1}{y} \\ x \cdot y & \text { if } y \geq 1 \text { and } y \geq \frac{1}{x} \\ \frac{1}{x \cdot y} & \text { otherwise. }\end{cases}
$$

They coincide in $\left[1, \infty\left[^{2}\right.\right.$ and in $[0,1]^{2}$.

It is interesting to note that in both examples there are some subsets of the cartesian power of the group where $E_{\mu}$ and $E^{\mu}$ coincide. In particular, in the second example, they coincide exactly in the positive and negative cones of $\mathbb{R}^{+}$considered as a totally ordered group. This opens the problem of finding conditions in which this result holds.

Example 7.3. In the plane $\mathbb{R}^{2}$ we can consider the fuzzy subgroup with respect to the Eukasiewicz $t$-norm $\mu$ defined by $\mu(x, y)=\min (1-|x|, 1-|y|)$ (a square pyramid with vertex in $(0,0,1))$. The associated invariant indistinguishability operator $E_{\mu}$ is $E_{\mu}\left((x, y),\left(x^{\prime}, y^{\prime}\right)\right)=\mu\left((x, y)-\left(x^{\prime}, y^{\prime}\right)\right)=\min (1-$ $\left.\left|x-x^{\prime}\right|, 1-\left|y-y^{\prime}\right|\right)$ and $E^{\mu}$ is $E^{\mu}\left((x, y),\left(x^{\prime}, y^{\prime}\right)\right)=1-\left|\mu(x, y)-\mu\left(x^{\prime}, y^{\prime}\right)\right|$. In the context of fuzzy mathematical morphology $\mu$ can be considered as a structural element.

\section{References}

[1] Boixader, D. Some properties concerning the quasi-inverse of a t-norm. Mathware \& Soft Computing 5, 1991, 5-12.

[2] Boixader, D., Mayor, G., Recasens, J. Aggregating Fuzzy Subgroups and T-vague subgroups AGOP 2017. Skövde (2017) 40-52.

[3] Bloch, I., Maître, H. Fuzzy mathematical morphologies: A comparative study, Pattern Recognition 28, 1995, 1341-1387.

[4] Carmona, N., Elorza, J., Recasens, J. Bragard, J. Permutable Fuzzy Consequence and Interior Operators and their Connection with Fuzzy Relations. Information Sciences 310 (2015) 36-51. 
[5] Demirci, M., Recasens, J. Fuzzy groups, fuzzy functions and fuzzy equivalence relations. Fuzzy Sets and Systems 144, 2004, 441-458.

[6] Formato, F., Gerla, G., Scarpati, L. Fuzzy subgroups and similarities. Soft Computing 3, 1999, 1-6.

[7] Fu-Gui Shi L-Fuzzy Relations and L-Fuzzy Subgroups. The Journal of Fuzzy Mathematics 8, 2000, 491-499.

[8] Mashour, A.S., Ghanim, M.H., Sidky, F.I. Normal fuzzy subgroups. Mat. Fak. Ser. Mat. 20, 1990, 53-59.

[9] Klement, E.P., Mesiar, R, Pap, E. Triangular Norms, Springer Netherlands, Dordrecht, 2000.

[10] Mordeson, J.N., Bhutani, K.R., Rosenfeld, A. Fuzzy Group Theory. Studies in Fuzziness and Soft Computing. Springer-Verlag, 2005.

[11] Recasens, J. Indistinguishability Operators. Modelling Fuzzy Equalities and Fuzzy Equivalence Relations. Studies in Fuzziness and Soft Computing. Springer, 2011.

[12] Recasens, J. Permutable Indistinguishability Operators, Perfect Vague Groups and Fuzzy Subgroups. Information Sciences 196 (2012), 129-142.

[13] Rosenfeld, A. Fuzzy subgroups. J. Math. Anal. and Applications 35, 1971, 512-517.

[14] Valverde, L. On the Structure of F-indistinguishability Operators. Fuzzy Sets and Systems 17, 1985, 313-328.

[15] Zadeh. L.A. Similarity relations and fuzzy orderings. Inform. Sci. 3, 1971, $177-200$. 